\title{
A identificação das necessidades das famílias como base para promoção de um programa de intervenção precoce
}

Identifying the needs of families as a basis for promoting an early intervention program

Identificar las necesidades de las familias como base para promover un programa de atención temprana

\author{
Vitor Franco \\ Professor doutor na Universidade de Évora, Évora, Portugal \\ E-mail: vfranco@uevora.pt ORCID: https://orcid.org/0000-0001-6510-6146 \\ Jaylsan Bandeira Fernandes de Castro \\ Mestra em Psicologia pela Universidade de Évora, Évora, Portugal \\ E-mail: jayfercastro@hotmail.com ORCID: https://orcid.org/0000-0001-9596-1522
}

Recebido em 27 de agosto de 2021

Aprovado em 24 de setembro de 2021

Publicado em 27 de dezembro de 2021

\section{RESUMO}

Os programas de intervenção precoce com crianças com deficiência evoluíram de uma perspectiva de estimulação, assente no trabalho terapêutico individual, para uma abordagem centrada na família. A implementação de serviços deste tipo implica um claro conhecimento das necessidades das famílias e suas características, pelo que o primeiro passo deverá ser a respectiva avaliação. Neste estudo procuramos identificar as necessidades das famílias de crianças com deficiência, assistidas na Associação dos Amigos e Pais de Pessoas Especiais, em Maceió, Brasil. Participaram 28 famílias e os dados foram coletados através de dois questionários que avaliam a forma como os pais identificam as necessidades da criança e deles mesmos. Os resultados mostram que o que mais inquieta as famílias se relaciona com o futuro, a saúde e o desenvolvimento da criança. Em geral, têm dificuldade em saber o que fazer para promover o desenvolvimento, não conhecem como ele ocorre, sabem pouco sobre opções de serviços e recursos disponíveis e registam pouca participação social. Globalmente as necessidades de informação são as mais valorizadas, assim como necessidades financeiras e a falta de acesso a serviços na comunidade. A falta de informação é especialmente impeditiva do seu envolvimento e empoderamento. Estes resultados apontam para a importância de conceber serviços e programas que respondam às necessidades das famílias e que possam ser efetivamente eficazes para as crianças. A identificação das necessidades e resultados esperados também permitirá avaliar posteriormente o impacto da intervenção e assim contribuir para o percurso escolar inclusivo das crianças.

Palavras-chave: Intervenção Precoce; Transtorno do desenvolvimento; Família. 
http://dx.doi.org/10.5902/1984686X67416

\section{ABSTRACT}

Early intervention programs with children with disabilities evolved from a stimulation perspective, based on individual therapeutic work, to a family-centered approach. The implementation of this type of services implies a clear knowledge about the needs of families and their characteristics, so the first step should be their assessment. In this study, we sought to identify the needs of families of children with disabilities, assisted by the Association of Friends and Parents of Special People (AAPPE) in Maceió, Brazil. Twentyeight families participated and data were collected through two questionnaires that assess how parents identify the needs of the child and themselves. The results show that what most worries families is child's future, health and development. In general, they find it difficult to know what to do to promote development, they do not know how it occurs, they know little about the options for services and resources available, and they register low social participation. Globally, information needs are the most valued, as are financial needs and lack of access to services in the community. Lack of information is a main obstacle to their involvement and empowerment. These results point to the importance of designing services and programs that respond to the needs of families and that can be effective for children. The identification of the expected needs and outcomes will also make it possible to subsequently assess the impact of the intervention and thus contribute to the children's inclusive educational trajectory.

Keywords: Early intervention; Disability; Family.

\section{RESUMEN}

Los programas de atención temprana para niños con discapacidad evolucionaron desde una perspectiva de estimulación, basada en el trabajo terapéutico individual, hacia un enfoque centrado en la familia. La implementación de servicios de este tipo implica un conocimiento claro de las necesidades de las familias y sus características, por lo que el primer paso debe ser su evaluación. En este estudio, buscamos identificar las necesidades de las familias de niños con discapacidad, asistidos por la Asociación de Amigos y Padres de Personas Especiales (AAPPE) en Maceió, Brasil. Participaron 28 familias y se recopilaron datos a través de dos cuestionarios que evalúan cómo los padres identifican las necesidades del niño y de ellos mismos. Los resultados muestran que lo que más preocupa a las familias está relacionado con el futuro, la salud y el desarrollo del niño. En general, les resulta difícil saber qué hacer para promover el desarrollo, no saben cómo ocurre, conocen poco sobre las opciones de servicios y recursos disponibles y registran baja participación social. Globalmente, las necesidades de información son las más valoradas, al igual que las necesidades financieras y la falta de acceso a los servicios en la comunidad. La falta de información es un obstáculo importante para su participación y empoderamiento. Estos resultados apuntan a la importancia de diseñar servicios y programas que respondan a las necesidades de las familias y que puedan ser efectivos para los niños. La identificación de las necesidades y resultados esperados también permitirá evaluar posteriormente el impacto de la intervención y contribuir así a la trayectoria educativa inclusiva de los niños.

Palabras clave: Atención temprana; Discapacidad; Familia. 
http://dx.doi.org/10.5902/1984686X67416

\section{Introdução}

\section{A intervenção precoce na promoção da inclusão}

O impacto das respostas educacionais dirigidas às crianças com transtornos do desenvolvimento, quando da sua entrada na vida escolar, depende absolutamente da qualidade do seu percurso inclusivo até então. A inclusão não pode ser concebida como relativa a uma prática, mas antes como um percurso no qual, desde o nascimento e ao longo de toda a vida, as crianças se confrontam com fatores facilitadores, promotores de inclusão, e com barreiras e obstáculos que a empurram para a exclusão (FRANCO, 2005). Por isso, a inclusão escolar é precedida de um conjunto de outros desafios inclusivos, etapas sem as quais dificilmente pode acontecer, ou pelo menos ter a eficácia pretendida. Este percurso inclusivo começa na inclusão familiar, ou seja, a forma como os pais vão adaptar o seu desenvolvimento à nova condição de terem um filho com transtorno do desenvolvimento. Passa depois pela intervenção nos primeiros tempos de vida e nas idades pré-escolares, a que chamamos intervenção precoce, e completa-se na inclusão social em todas as suas dimensões (relações, cultura, desporto, vida social) e na inclusão profissional e promoção da autonomia pessoal.

Sem uma intervenção adequada nos primeiros tempos de vida, a inclusão educativa está ameaçada e posta em causa. A Intervenção Precoce (IP) corresponde a um conjunto ou sistema de apoios ao desenvolvimento da criança com transtorno do desenvolvimento, ou em risco de os vir a apresentar, que tem como objetivos finais promover o máximo desenvolvimento da criança e assegurar a sua máxima inclusão. Podemos considerar que a IP é, hoje em dia, uma condição fundamental para a eficácia de qualquer processo de intervenção educativa escolar, pois é ela que vai permitir tanto assegurar as melhores condições de desenvolvimento como fomentar envolvimento e inclusão por parte da família e do contexto (FRANCO et.al, 2017).

\section{Modelo de intervenção precoce centrada na família}

A IP é uma área de intervenção e investigação em que, nas últimas décadas, e por influência das perspectivas ecológicas e sistêmicas, tem ocorrido uma verdadeira revolução, quer em termos da sua filosofia, quer nas práticas, com a ultrapassagem do modelo médico tradicional (PIMENTEL, 2005; COUTINHO, 2004). A IP tem-se distanciado de uma abordagem centrada na criança e no problema, para adotar uma perspectiva mais 
http://dx.doi.org/10.5902/1984686X67416

alargada, considerando o desenvolvimento de toda a família, nos seus múltiplos aspectos (FRANCO \& APOLÓNIO, 2008; CARDOSO, SEIXAS \& PISCALHO, 2017). Chegamos assim a uma perspectiva "centrada na família" na Intervenção Precoce na Infância, que pretende que a família seja capaz de decidir sobre o seu futuro e o da sua criança e de agir de forma adequada às suas necessidades (FRANCO, 2009). Esta nova abordagem implica múltiplas mudanças ao nível do trabalho com as crianças com transtornos do desenvolvimento (PIMENTEL, 2004).

DUNST, TRIVETTE e DEAL (1988) contribuíram significativamente para a reformulação dos serviços de atendimento às famílias e crianças em IP (MCWILLIAM, YOUNG \& HARVILLE, 1996; CORREIA \& SERRANO, 2002; CRUZ, FONTES, \& CARVALHO, 2003), tendo subjacente este modelo de intervenção centrado na família, fazendo sobressair os conceitos de "empowerment" e "enabling", sublinhando a característica diferenciadora destas estratégias e práticas que é a ênfase na dimensão contextual, entendida como uma fonte de oportunidades e de experiências fundamentais para o desenvolvimento da criança (DUNST, 1985, 2000).

A implementação de um modelo centrado na família é orientada por um conjunto de pressupostos que visam a co-responsabilização, capacitação e fortalecimento das famílias (CRUZ, FONTES, \& CARVALHO, 2003). Trata-se de uma abordagem holística e multisectorial para a prestação de serviços, em que os pontos fortes em conjunto com os recursos visam prover as necessidades de cada família, formando a base para um modelo individualizado e dinâmico de cuidado (SIEBES, KETELAAR, GORTER, ALSEM \& JONGMANS, 2012). Estas práticas participativas incluem envolvimento ativo e informado dos pais nas tomadas de decisão, usar os pontos fortes e habilidades existentes, bem como o desenvolvimento de novas capacidades necessárias para obter recursos, suporte e orientação.

Nesta perspectiva, os pais desempenham um papel fundamental na promoção do desenvolvimento precoce dos seus filhos e a responsividade parental é considerada uma das principais qualidades parentais para a promoção da aprendizagem e desenvolvimento da criança e representa uma influência decisiva no desenvolvimento e bem-estar sócioemocional das crianças com deficiência (MAHONEY \& PERALES, 2013).

Diferentes estudos (BAILEY, 1991; CRUZ, FONTES, \& CARVALHO, 2003) mostram que a participação da família no processo de intervenção é um elemento fundamental para uma visão mais ampla e uma melhor percepção do problema, e a IP tem procurado 
http://dx.doi.org/10.5902/1984686X67416

identificar conjuntos de serviços, apoios e recursos necessários para responder quer às necessidades específicas de cada criança, quer às necessidades das suas famílias quanto à promoção do desenvolvimento da criança (DUNST, 1988; BAILEY \& WOLERY 1992; PIMENTEL, 2004). Cabe à família identificar quais são os contextos naturais da sua vida, e da criança, em que devem ocorrer experiências de aprendizagem que sejam ricas e significativas e que promovam ao máximo o desenvolvimento.

O denominado modelo de terceira geração da IP se estabelece em quatro componentes essenciais: a) as oportunidades de aprendizagem da criança; b) o apoio às competências dos pais; c) os recursos da família ou da comunidade envolvente; e d) as práticas de ajuda centradas na família. Conjuntamente visam assegurar que as experiências e oportunidades proporcionadas às famílias e crianças as estimulem e promovam as suas capacidades e, ainda, que os profissionais conduzam as intervenções de uma forma consistente com o quadro de referência proposto (ALMEIDA, 2011; SOUSA \& PISCALHO, 2016).

A eficácia dos programas de IP está, assim, diretamente dependente da forma como a família se envolve no processo de intervenção, das suas percepções e dos seus sentimentos (CORREIA \& SERRANO, 2002). Envolver os pais no processo de intervenção é conduzir a que adquiram capacidades que promovam o desenvolvimento da criança. De igual modo, o envolvimento no sistema de recursos, a informação, as atividades de ensino para a família, o apoio pessoal e familiar e a orientação, vão influenciar diretamente as competências da família ao nível do seu funcionamento e as capacidades parentais para enfrentar a situação (PIMENTEL, 2004).

Teremos assim um modelo de intervenção baseado nas forças e competências da criança e da família, em que o profissional se torna um recurso e um apoio, responsável por ajudar a família a identificar os objetivos da intervenção e fornecer informações que permitam a tomada de decisão, contribuindo para a sua autonomia (SOUSA \& PISCALHO, 2016), sendo a identificação das necessidades um dos pontos fulcrais nas práticas de intervenção centradas na família, em função das prioridades estabelecidas para responder adequadamente à realidade familiar.

\section{As necessidades da família e a criação de repostas de intervenção}

As necessidades das famílias de crianças com deficiência são múltiplas e diversas, variando em função de fatores como: o tipo da deficiência; a idade da criança; o momento 
http://dx.doi.org/10.5902/1984686X67416

evolutivo do casal; e as condições pessoais, económicas, sociais e culturais que afetam direta ou indiretamente o contexto familiar (GINÉ, 2000; RAMIREZ, 2015). No processo de identificação dessas necessidades, o profissional deve ir ao encontro da família promovendo um relacionamento produtivo e de confiança (ESPE-SHERWINDT, 2008) ajudando-a a transformar as suas preocupações em necessidades, ou seja, esclarecendoas de modo a identificar recursos adequados a essas necessidades (SIMÕES \& MESQUITA, 2010). Partindo do levantamento das necessidades das famílias, é então possível identificar os recursos necessários e estabelecer estratégias centradas na família como, por exemplo, redes de apoio social ou promoção de práticas de adaptação e resposta à sua realidade (DIAS \& PIMENTEL, 2017). Perceber quais as necessidades que as famílias apresentam e com que tipos de apoio podem contar será a melhor forma de planejar possíveis respostas (SIMÕES \& MESQUITA, 2010), daí que a identificação dessas necessidades tenha um papel decisivo no início do processo da intervenção precoce (DUNST, 1988; ESPE-SHERWINDT, 2008).

A identificação das necessidades das famílias deve ser entendida assim como uma componente básica do processo de $\mathrm{IP}$, antes de qualquer decisão sobre a intervenção (BJORCK-ALKESSON, GRANLUND \& SIMEONSSON, 2000; GINÉ, 2000). Através da identificação das necessidades, objetivos e prioridades das famílias, podem ajustar-se os apoios, sociais, educativos ou terapêuticos existentes e modificar o funcionamento enquanto sistema (GONÇALVES \& SIMÕES, 2010).

RAMIREZ (2015) propõe a organização das necessidades das famílias em quatro categorias: necessidades cognitivas, necessidades emocionais, necessidades sociais, e necessidades materiais e de recursos.

Bailey e Simeonsson (1988), por seu lado, agrupam as necessidades das famílias de crianças com deficiência em cinco categorias:

1) Necessidades de Informação, que se referem ao conjunto de informações sobre os serviços de que os filhos poderão beneficiar no presente e no futuro. Sendo a deficiência uma situação nova e inesperada, as famílias precisam compreender melhor a situação e a condição da criança (PAVÃO, GUALDA, CIA, SANTOS \& CHRISTOVAM, 2018) e carecem de informação que facilite o processo de adaptação à nova realidade que vivem (FRANCO, 2005; GUALDA, BORGES \& CIA, 2013).

2) Necessidades de Apoio, que dizem respeito aos recursos formais e informais que ajudarão a diminuir o estresse familiar. Este apoio abrange toda a rede social (formal e 
http://dx.doi.org/10.5902/1984686X67416

informal), que afeta a família. As redes de apoio formais englobam profissionais (e.g., médicos, enfermeiros, terapeutas e outros profissionais da área da saúde, educação e social) enquanto que as redes de apoio informal são constituídas por membros de família alargada, amigos, vizinhos, colegas de trabalho ou grupos sociais (e.g., instituições religiosas, clubes e organizações voluntárias), que proporcionam e potenciam as competências fundamentais para a família na situação de crise (SERRANO, 2008).

3) Necessidades de Explicar aos Outros, que inclui a inquietação dos pais em ter de explicar a situação do seu filho às outras pessoas, fazendo-as compreender a nova situação que vivenciam. A família irá confrontar-se com ideias pré-concebidas, terá de lidar com a curiosidade e com perguntas muitas vezes inadequadas relacionadas com a criança, que ativam a vulnerabilidade da família exposta a invasões de privacidade e aos efeitos da curiosidade alheia no seu quotidiano (SÁ \& RABINOVICH, 2006). Por isso há também uma grande dificuldade na forma como os pais vão explicar a condição do seu filho aos familiares, vizinhos, amigos, ou outras pessoas (SIMÕES \& MESQUITA, 2010).

4) Necessidades de Acesso aos serviços da comunidade. Os pais necessitam conhecer os serviços da comunidade e a forma de obterem os seus benefícios; Têm, naturalmente, fortes inquietações relativas aos serviços e necessitam conhecer os serviços existentes e como ter acesso a eles, quais os programas de que dispõem para responder aos seus problemas, ao nível da educação, saúde, reabilitação e apoio social, bem como os seus direitos e benefícios sociais (CORREIA \& SERRANO, 2002; BAILEY et al., 2011).

5) Necessidades Financeiras e de Funcionamento familiar, que se referem às necessidades primárias e aos custos de serviços. A família com uma pessoa com deficiência sofre mais perdas econômicas e tem de enfrentar custos adicionais derivados da deficiência (RAMIREZ, 2015). Turnbull \& Turnbull (1990), na sua categorização das necessidades familiares mais específicas, salientam um conjunto de alterações ao funcionamento da família que surgem com a problemática da criança, carregando inquietações e responsabilidades a vários níveis, incluindo as necessidades económicas. A deficiência da criança pode levar ao agravamento de despesas do agregado familiar, seja por necessidade de adquirir equipamento especializado ou intervenções específicas (SAMPAIO \& GERALDES, 2006). Outro dos motivos que contribui para agravar a situação financeira é um dos progenitores ter de abandonar o emprego para cuidar da criança o tempo inteiro, reduzindo ainda mais o rendimento (MORGANHO, 2015). 
http://dx.doi.org/10.5902/1984686X67416

Em síntese, as práticas da avaliação e intervenção centradas na família devem ter em linha de conta tanto a identificação das necessidades da família, como a localização dos recursos e apoios que permitam: 1) dar resposta a essas necessidades; 2) ajudar as famílias a utilizar as suas competências; e 3) capacitar a família a adquirir novas competências, de forma a mobilizar recursos para que consiga satisfazer as suas necessidades (DUNST, TRIVETTE \& DEAL, 1994).

A criação de serviços de intervenção precoce centrados na família, dentro de um modelo atual, exige que se comece por conhecer as necessidades das famílias em cada contexto (CORREIA \& SERRANO, 2002). Daí que neste estudo pretendamos identificar as necessidades das famílias de crianças com deficiência ou transtornos do desenvolvimento, com 6 anos ou menos, que buscam assistência na Associação dos Amigos e Pais de Pessoas Especiais (AAPPE).

\section{Objetivos e contexto}

A Associação dos Amigos e Pais de Pessoas Especiais é uma associação fundada em 1987 e certificada em 1995 como Entidade Beneficente de Assistência Social, tendo sede em Maceió, mas sendo reconhecida a nível nacional pelas entidades que atuam na área da proteção social. Em 2016, foi inserida na Rede Nacional de Primeira Infância, marco fundamental para a Instituição, que tem como público prioritário as crianças e adolescentes com múltiplas deficiências do Estado de Alagoas. A AAPPE possui três unidades de atendimento na área de saúde na cidade Maceió: o CER III Centro Especializado de Reabilitação Intelectual, Física e Auditiva; o Programa de Reabilitação Neurossensorial; e a Oficina Ortopédica. Os serviços foram expandidos para os municípios de Santana do Ipanema, reconhecido em 2016 como Centro Especializado em Reabilitação Neurossensorial e Auditiva - CER II, e município de Penedo, habilitado com o Programa de Reabilitação Neurossensorial.

Face à intenção de desenvolver e modernizar os seus serviços de Intervenção Precoce e visando a criação de uma nova resposta na cidade de Maceió, entendeu que o primeiro passo deveria ser conhecer as famílias e as suas necessidades, de modo a adequar as respostas a implementar numa perspectiva ecológica e centrada na família.

Neste estudo, de caráter exploratório, procuram-se identificar as necessidades sentidas pelas famílias de crianças que procuraram apoio nos serviços da saúde da 
http://dx.doi.org/10.5902/1984686X67416

AAPPE. Dessa forma contribuindo também para a criação de respostas específicas e para melhoria da qualidade dos serviços prestados a essas famílias.

São objetivos específicos: a) identificar o que famílias respondem às necessidades das suas crianças com transtornos do desenvolvimento; b) identificar as principais necessidades das famílias; e c) verificar a influência de diferentes variáveis sociodemográficas na forma como as famílias identificam as suas necessidades.

\section{Metodologia}

Para alcançarmos estes objetivos foi organizado um estudo exploratório e qualitativo, em que foram usados questionários para conhecer opiniões de pais e mães sobre a problemática em estudo.

\section{Participantes}

Participaram neste estudo 28 famílias de crianças com deficiência (27 mães e 21 pais) de crianças até aos 6 anos, que frequentam os serviços de saúde da AAPPE.

As mães possuem idades entre 20 e 68 anos (média de 34.96) e os pais entre 25 e 66 anos (média de 40.43). 64.3\% dos participantes são casados ou em união de facto e $14.3 \%$ são famílias monoparentais.

Relativamente à formação académica das mães, 25\% têm Ensino Superior, 28.6\% têm Ensino Médio, 10.7\% Fundamental, e 17.9\% não possuem qualquer grau de ensino. Quanto aos pais, 25\% têm Ensino Superior, 21.4\% têm Ensino Médio, 7,1\% Fundamental, $21.5 \%$ não tem qualquer grau concluído.

Quanto à situação profissional, 53.6\% das mães são empregadas domésticas, 10.7\% encontram-se desempregadas e 10,8 \% têm profissões especializadas. Em relação aos pais $10.7 \%$ são trabalhadores autónomos e outros $10.7 \%$ comerciantes, enquanto as restantes profissões se encontram muito dispersas.

No que se refere às crianças, têm entre 11 meses e 6 anos (média de 3.76 anos). As principais deficiências reportadas quanto ao diagnóstico são: a deficiência intelectual (39.3\%); transtornos na fala e linguagem (35.7\%); deficiência motora (32.1\%); e 28,6\% Transtorno do Espetro do Autismo e Atraso do desenvolvimento. Com porcentagem baixa estão as crianças com deficiência visual, auditiva e multideficiência (cada uma com 3.6\%). $37.5 \%$ das crianças frequentam Escola Básica, 28.6\% a Creche, e 21.4\% não frequentam qualquer estabelecimento de ensino. 
http://dx.doi.org/10.5902/1984686X67416

\section{Instrumentos}

Para a recolha de dados relativos às necessidades das famílias foram utilizados dois instrumentos: "Inquérito aos Resultados na Família (IRF)" e "Questionário Identificação das Necessidades das Famílias de Crianças com Deficiência" (QINF).

O "Inquérito aos Resultados na Família", versão 2012 do Family Outcome Survey (FOS), foi desenvolvido pelo Early Childhood Outcomes Center (ECO) (BAILEY et al. 2008) e centra-se na forma como as famílias se sentem capazes de responder às necessidades do seu filho. É composto por 24 itens, sendo pedido aos pais que selecionem para cada um a opção que melhor descreve a sua família neste momento, numa escala de tipo Likert de cinco pontos. Os itens estão agrupados em cinco sub-escalas: 1 - Compreender os pontos fortes, as necessidades e as capacidades do seu filho (4 itens); 2 - Conhecer os seus direitos e defender os interesses do seu filho (5 itens); 3 - Ajudar o seu filho a desenvolverse e a aprender (4 itens); 4- Formar sistemas de apoio; 5 - Ter acesso à comunidade (6 itens). Este instrumento foi construído com base numa perspectiva de IP centrada na família, pretendendo avaliar os resultados obtidos ao nível desta.

O "Questionário de Identificação das Necessidades das Famílias de Crianças com Deficiência" consiste numa lista abrangente de necessidades, dificuldades e problemas vividos pela família (FRANCO \& APOLÓNIO, 2002; MEDINA-GOMEZ \& FRANCO, 2018) de forma a que cada pai/mãe possa identificar os que sentem como mais importantes. Numa primeira parte foram colhidas as informações sociodemográficas dos participantes e também da criança. O questionário é composto por 50 itens de autorresposta, numa escala de cinco pontos entre nada (1) e muitíssimo (5).

\section{Procedimentos e questões éticas}

O estudo foi autorizado pelo Conselho Cientifico da ECS da Universidade de Évora, que tutelou a realização da pesquisa. Num primeiro momento, os participantes foram esclarecidos acerca dos objetivos e procedimentos da pesquisa, e da possibilidade de terem acesso, posteriormente, aos resultados do estudo. Foi solicitado a cada mãe e cada pai a sua participação voluntária e um termo de Consentimento Informado. Foi garantido o anonimato, o respeito pela confidencialidade, e respeitados os procedimentos éticos aplicáveis.

A aplicação dos instrumentos foi realizada pelos técnicos da AAPPE junto dos pais, mães e/ou cuidadores de crianças que frequentam a Unidade de Saúde da AAPPE. 


\section{Resultados: apresentação e discussão}

Serão apresentados de seguida os resultados obtidos em cada um dos instrumentos e também os principais resultados das análises efetuadas, tendo consideração às características sociodemográficas dos participantes.

\section{Competências e resultados da família}

$\mathrm{Na}$ Tabela 1 podemos ver o conjunto completo dos resultados obtidos nos itens do IRF. Tendo sido pedido aos participantes que identificassem a forma como avaliam as suas competências, vemos que se consideram mais competentes nos seguintes itens: "Somos capazes de dizer quando o nosso filho está fazendo progressos/evoluindo." ( $M=3.93$ ); "As nossas necessidades de alimentação, de vestuário ou com a casa estão satisfeitas." ( $M=3.85)$; "Compreendemos os atrasos e/ou necessidades do nosso filho." ( $M=3.81$ ) e "Temos amigos ou familiares em quem podemos confiar quando precisamos de ajuda." $(M=3.70)$.

Tabela 1 - Resultados do Inquérito aos Resultados na Família

(continua)

\begin{tabular}{|c|c|c|c|c|c|c|c|}
\hline Itens & Média & d.p. & $\frac{\pi}{\frac{\pi}{0}}$ & \begin{tabular}{l} 
: \\
\hdashline 0 \\
0
\end{tabular} & $\frac{\circ}{\frac{O}{4}}$ & $\stackrel{\frac{0}{5}}{\frac{1}{2}}$ & $\begin{array}{l}\overline{0} \\
\bar{\varepsilon} \\
\dot{0}\end{array}$ \\
\hline $\begin{array}{l}\text { Sabemos quais são os próximos } \\
\text { passos no crescimento e } \\
\text { aprendizagem do nosso filho. }\end{array}$ & 2.36 & 1.13 & $17.9 \%$ & $53.6 \%$ & $10.7 \%$ & $10.7 \%$ & $7.1 \%$ \\
\hline $\begin{array}{l}\text { Compreendemos os pontos fortes e } \\
\text { as capacidades do nosso filho }\end{array}$ & 3.37 & 1.04 & - & $28.6 \%$ & $14.3 \%$ & $42.9 \%$ & $10.7 \%$ \\
\hline $\begin{array}{l}\text { Compreendemos os atrasos e/ou } \\
\text { necessidades do nosso filho. }\end{array}$ & 3.81 & 1.18 & $7.1 \%$ & $7.1 \%$ & $10.7 \%$ & $42.9 \%$ & $28.6 \%$ \\
\hline $\begin{array}{l}\text { Somos capazes de dizer quando o } \\
\text { nosso filho está fazendo } \\
\text { progressos/evoluindo. }\end{array}$ & 3.93 & 0.92 & $3.6 \%$ & $3.6 \%$ & $10.7 \%$ & $57.1 \%$ & $21.4 \%$ \\
\hline $\begin{array}{l}\text { Conseguimos encontrar e utilizar os } \\
\text { apoios, serviços e programas que } \\
\text { nos são disponibilizados. }\end{array}$ & 2.96 & 1.23 & $7.1 \%$ & $42.9 \%$ & $7.1 \%$ & $32.1 \%$ & $10.7 \%$ \\
\hline $\begin{array}{l}\text { Sabemos os nossos direitos em } \\
\text { relação às necessidades especiais } \\
\text { do nosso filho. }\end{array}$ & 2.92 & 1.35 & $10.7 \%$ & $35.7 \%$ & $14.3 \%$ & $14.3 \%$ & $17.9 \%$ \\
\hline $\begin{array}{l}\text { Sabemos quem contactar e o que } \\
\text { fazer quando temos questões ou } \\
\text { dúvidas. }\end{array}$ & 3.00 & 1.30 & $10.7 \%$ & $28.6 \%$ & $17.9 \%$ & $21.4 \%$ & $14.3 \%$ \\
\hline $\begin{array}{l}\text { Sabemos que opções estão } \\
\text { disponíveis quando o nosso filho } \\
\text { deixa o programa. }\end{array}$ & 2.31 & 1.26 & $32.1 \%$ & $25.0 \%$ & $14.3 \%$ & $17.9 \%$ & $3.6 \%$ \\
\hline $\begin{array}{l}\text { Nos sentimos confortáveis para } \\
\text { pedir os serviços ou apoios que o } \\
\text { nosso filho ou família necessitam. }\end{array}$ & 3.15 & 1.16 & $3.6 \%$ & $32.1 \%$ & $14.3 \%$ & $32.1 \%$ & $10.7 \%$ \\
\hline
\end{tabular}


http://dx.doi.org/10.5902/1984686X67416

Tabela 1 - Resultados do Inquérito aos Resultados na Família

(conclusão)

\begin{tabular}{|c|c|c|c|c|c|c|c|}
\hline tens & Média & d.p. & $\begin{array}{l}\frac{\pi}{5} \\
\frac{\pi}{2}\end{array}$ & $\begin{array}{l}0 \\
\text { O } \\
\text { o }\end{array}$ & $\frac{8}{8}$ & $\stackrel{0}{\stackrel{0}{3}}$ & $\begin{array}{l}\bar{\alpha} \\
\text { है } \\
\text { ঠ̊ }\end{array}$ \\
\hline $\begin{array}{l}\text { Conseguimos ajudar o nosso filho a } \\
\text { relacionar-se bem com outras } \\
\text { pessoas. }\end{array}$ & 3.32 & 1.12 & $3.6 \%$ & $28.6 \%$ & $10.7 \%$ & $46.4 \%$ & $10.7 \%$ \\
\hline $\begin{array}{l}\text { Conseguimos ajudar o nosso filho a } \\
\text { aprender novas competências. }\end{array}$ & 3.44 & 1.01 & $3.6 \%$ & $10.7 \%$ & $35.7 \%$ & $32.1 \%$ & $14.3 \%$ \\
\hline $\begin{array}{l}\text { Conseguimos ajudar o nosso filho a } \\
\text { satisfazer as suas necessidades. }\end{array}$ & 3.56 & 0.93 & - & $17.9 \%$ & $17.9 \%$ & $50.0 \%$ & $10.7 \%$ \\
\hline $\begin{array}{l}\text { Conseguimos trabalhar nos } \\
\text { objetivos do nosso filho durante as } \\
\text { nossas rotinas quotidianas. }\end{array}$ & 2.93 & 1.07 & $3.6 \%$ & $39.3 \%$ & $21.4 \%$ & $25.0 \%$ & $7.1 \%$ \\
\hline $\begin{array}{l}\text { Nos sentimos confortáveis ao falar } \\
\text { com a família e amigos acerca das } \\
\text { necessidades do nosso filho. }\end{array}$ & 3.43 & 1.37 & $10.7 \%$ & $21.4 \%$ & $7.1 \%$ & $35.7 \%$ & $25.0 \%$ \\
\hline $\begin{array}{l}\text { Temos amigos ou familiares que nos } \\
\text { ouvem e mostram interesse. }\end{array}$ & 3.33 & 1.41 & $14.3 \%$ & $14.3 \%$ & $17.9 \%$ & $25.0 \%$ & $25.0 \%$ \\
\hline $\begin{array}{l}\text { Temos oportunidade de falar com } \\
\text { outras famílias que têm crianças } \\
\text { com necessidades semelhantes. }\end{array}$ & 3.63 & 1.25 & - & $28.6 \%$ & $10.7 \%$ & $25.0 \%$ & $32.1 \%$ \\
\hline $\begin{array}{l}\text { Temos amigos ou familiares em } \\
\text { quem podemos confiar quando } \\
\text { precisamos de ajuda. }\end{array}$ & 3.70 & 1.24 & $3.6 \%$ & $17.9 \%$ & $14.3 \%$ & $28.6 \%$ & $32.1 \%$ \\
\hline $\begin{array}{l}\text { Consigo satisfazer as minhas } \\
\text { próprias necessidades e faço coisas } \\
\text { de que gosto. }\end{array}$ & 2.85 & 1.20 & $10.7 \%$ & $35.7 \%$ & $14.3 \%$ & $28.6 \%$ & $7.1 \%$ \\
\hline $\begin{array}{l}\text { O nosso filho participa em atividades } \\
\text { sociais, recreativas ou religiosas que } \\
\text { queremos. }\end{array}$ & 2.57 & 1.29 & $21.4 \%$ & $39.3 \%$ & $7.1 \%$ & $25.0 \%$ & $7.1 \%$ \\
\hline $\begin{array}{l}\text { Como família, fazemos coisas juntos } \\
\text { de que gostamos. }\end{array}$ & 3.56 & 1.25 & $3.6 \%$ & $25.0 \%$ & $7.1 \%$ & $35.7 \%$ & $25.0 \%$ \\
\hline $\begin{array}{l}\text { As nossas necessidades médicas e } \\
\text { dentárias estão satisfeitas. }\end{array}$ & 3.27 & 1.46 & $14.3 \%$ & $17.9 \%$ & $14.3 \%$ & $21.4 \%$ & $25.0 \%$ \\
\hline $\begin{array}{l}\text { As necessidades de cuidados do } \\
\text { nosso filho estão satisfeitas. }\end{array}$ & 3.62 & 1.17 & $7.1 \%$ & $10.7 \%$ & $10.7 \%$ & $46.4 \%$ & $17.9 \%$ \\
\hline $\begin{array}{l}\text { As nossas necessidades de } \\
\text { transporte estão satisfeitas. }\end{array}$ & 3.08 & 1.47 & $17.9 \%$ & $17.9 \%$ & $17.9 \%$ & $17.9 \%$ & $21.4 \%$ \\
\hline $\begin{array}{l}\text { As nossas necessidades de } \\
\text { alimentação, de vestuário ou com a } \\
\text { casa estão satisfeitas. }\end{array}$ & 3.85 & 0.95 & - & $10.7 \%$ & $17.9 \%$ & $42.9 \%$ & $25.0 \%$ \\
\hline
\end{tabular}

Fonte: Os autores (2020).

Por outro lado, os pais consideram obter piores resultados no que diz respeito a: "Consigo satisfazer as minhas próprias necessidades e faço coisas de que gosto." $(M=2.85)$; "O nosso filho participa em atividades sociais, recreativas ou religiosas que queremos." (M=2.57); "Sabemos quais são os próximos passos no crescimento e aprendizagem do nosso filho." (M=2.36); e "Sabemos que opções estão disponíveis quando o nosso filho deixar o programa." (M=2.31). 
http://dx.doi.org/10.5902/1984686X67416

Analisando os resultados de acordo com a dimensão/ subescala (Tabela 2), verificase que cada uma destas possui um elevado índice de consistência interna (alfa de Cronbach entre 0.735 e 0.854 ). Os pais/mães consideram apoiar mais os seus filhos nas subescalas "compreender os pontos fortes, as necessidades e as capacidades do seu filho

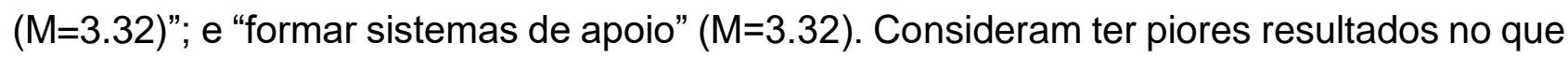
diz respeito ao "conhecer os seus direitos e defender os interesses do seu filho" $(M=2.85)$.

Tabela 2 - Resultados das Subescalas do IRF

\begin{tabular}{lccc}
\hline \multicolumn{1}{c}{ Subescalas } & Média & d.p. & $\begin{array}{c}\text { Alfa de } \\
\text { Cronbach }\end{array}$ \\
\hline $\begin{array}{l}\text { Compreender os pontos fortes, as necessidades e as } \\
\text { capacidades do seu filho }\end{array}$ & 3.32 & 0.83 & 0.735 \\
\hline Conhecer os direitos e defender interesses do seu filho & 2.85 & 0.94 & 0.822 \\
\hline Ajudar o seu filho a se desenvolver e a aprender & 3.28 & 0.81 & 0.752 \\
\hline Formar sistemas de apoio & 3.32 & 1.05 & 0.803 \\
\hline Ter acesso à comunidade & 3.25 & 1.02 & 0.854 \\
\hline
\end{tabular}

Fonte: Os autores (2020).

Como vimos, os pais/mães sentem-se menos capazes de apoiar os filhos quando terminam um ciclo ou programa de apoio, de antecipar e identificar o próximo passo no crescimento e aprendizagem da criança, e na promoção da participação nas atividades sociais, recreativas ou religiosas. Deste modo, podemos considerar que essas são as necessidades mais sentidas pelas famílias, e os domínios em que se sentem menos capazes de obter resultados.

Embora se sintam mais capazes de compreender os pontos fortes, as necessidades e as capacidades do seu filho e criar sistemas de apoio, o que pode constituir os pontos fortes e os recursos da família para a IP, os pais/mães sentem-se menos capazes de apoiar os filhos no que se refere a conhecer os seus direitos e defender os seus interesses.

Sendo esta a linha de base representativa da situação destas famílias, um programa de intervenção a implementar deve poder obter resultados ao nível de todas estas dimensões, especialmente daquelas em que os resultados são mais baixos.

\section{Necessidades das famílias}

Quanto às necessidades específicas que mais preocupam os pais/mães, observou-se que se relacionam mais com: "Saber como vai ser o futuro do seu filho/a quando Ihe faltar" 
http://dx.doi.org/10.5902/1984686X67416

(4.04); "Saúde da criança" (3.48); "Saber o que vai acontecer no futuro" (3.37) e "Saber o que fazer para estimular o desenvolvimento" (3.36).

Por outro lado, são menores as preocupações dos pais com: "Não ter afeto e aceitação" (1.68); "Conflitos com a família alargada" (1.68); "Conflito com os vizinhos" (1.54) e "Sentir vergonha da condição do filho/a" (1.00).

No sentido de obter uma perspectiva dimensional mais abrangente, agruparam-se os itens de acordo as categorias de necessidades referidas pelo Bailey e Simeonsson (1988) (Tabela 3).

Tabela 3 - Itens de necessidades das famílias agrupados em categorias

\begin{tabular}{|c|c|c|c|c|c|c|c|c|c|c|c|c|c|}
\hline Categorias & \multicolumn{13}{|c|}{ Itens } \\
\hline $\begin{array}{l}\text { Necessidades de informação } \\
\text { (13 itens) }\end{array}$ & 31 & 2 & 6 & 7 & 30 & 19 & 5 & 50 & 49 & 48 & 1 & 4 & 3 \\
\hline Necessidades de apoio (11 itens) & 36 & 18 & 26 & 20 & 40 & 35 & 37 & 42 & 47 & 34 & 41 & & \\
\hline $\begin{array}{l}\text { Necessidades de explicar aos } \\
\text { outros ( } 5 \text { itens) }\end{array}$ & 17 & 8 & 39 & 33 & 9 & & & & & & & & \\
\hline $\begin{array}{l}\text { Necessidades de serviços da } \\
\text { comunidade ( } 4 \text { itens) }\end{array}$ & 22 & 24 & 23 & 32 & & & & & & & & & \\
\hline Necessidades financeiras ( 7 itens) & 10 & 12 & 11 & 13 & 25 & 45 & 28 & & & & & & \\
\hline Funcionamento familiar ( 9 itens) & 14 & 21 & 38 & 43 & 44 & 16 & 15 & 46 & 27 & & & & \\
\hline
\end{tabular}

Fonte: Os autores (2020).

Cada uma destas dimensões mostrou um elevado índice de consistência interna (Alfa de Cronbach entre 0.691 e 0.888). Como se observa na Tabela 4, as necessidades que mais preocupam os pais são as necessidades de informação (2.89) e as financeiras (2.71). As que menos preocupam as famílias são as necessidades de explicar aos outros (1.91) e as relativas ao funcionamento familiar (1.97).

Tabela 4 - Agrupamento das necessidades das famílias

\begin{tabular}{lccc}
\hline \multicolumn{1}{c}{ Categorias } & Média & d.p. & Alfa de Cronbach \\
\hline Necessidades de informação & 2.88 & 0.81 & 0.873 \\
\hline Necessidades de apoio & 2.41 & 0.96 & 0.888 \\
\hline Necessidades de explicar aos outros & 1.91 & 0.84 & 0.691 \\
\hline Necessidades de serviços da comunidade & 2.41 & 1.08 & 0.789 \\
\hline Necessidades financeiras & 2.70 & 1.12 & 0.896 \\
\hline Funcionamento familiar & 1.97 & 1.03 & 0.888
\end{tabular}

Fonte: Os autores (2020). 
http://dx.doi.org/10.5902/1984686X67416

Comparando as necessidades que preocupam as famílias e as subescalas do IRF, verifica-se que as correlações entre ambas as variáveis são tendencialmente negativas, o que é expectável e significa que quanto mais as famílias conseguem apoiar os seus filhos, menos preocupação tem com as necessidades enunciadas.

As principais necessidades das famílias relacionam-se com o futuro, a saúde e o desenvolvimento da criança, sendo a preocupação com o futuro da criança a que mais sobressai. Estes resultados corroboram os estudos de Gallagher Beckman \& Cross (1983), Bailey \& Simeonsson (1988), Pereira (1996), Franco \& Apolónio (2002), Dias \& Pimentel (2017) Pavão, et al. (2018), que também apontam para ser esta a inquietação mais sentida pela família.

Relativamente às diferentes categorias das necessidades das famílias, os resultados apontam para a importância das de informação e financeiras. Resultados consistentes com o que diz a literatura nos estudos de Pereira (1996), Bailey, Blasco \& Simeonsson, (1992), Simões \& Mesquita (2010), Ramirez (2015) E Soqueiro, et al. (2017), que também apontam as necessidades de informação e financeiras como as que as famílias mais reportam.

Ao se considerar a relação entre a situação profissional das mães e as necessidades que as preocupam, as desempregadas e as que trabalham na função pública são as que apresentam mais necessidades de informação e financeiras. O que sugere que, independentemente da situação econômica e profissional, estas necessidades são prioritárias para as famílias, apesar de outros estudos (BAILEY, BLASCO \& SIMEONSSON, 1992; PEREIRA, 1996) relatarem que é evidente que as necessidades financeiras são maiores nas mães com mais baixa renda.

Analisando a relação entre a tipologia familiar e as dimensões das necessidades, os resultados apontam que as famílias reconstruídas e monoparentais reportam mais necessidades de informação. Embora Pereira (1996) refira que as famílias monoparentais são as que menos valorizam a necessidade de informação, verifica-se que aqui estas famílias apresentam necessidades em todas as categorias: informação, apoio, explicar aos outros, serviços da comunidade, financeiras e funcionamento familiar, sendo, no entanto, a necessidade de informação a mais relevante.

Quanto à relação entre tipologia familiar e as subescalas do IRF, os resultados apontam que as famílias de pais casados ou em união de fato tendem a sentir-se mais competentes no apoio aos filhos em todas as subescalas. 
http://dx.doi.org/10.5902/1984686X67416

$\mathrm{Na}$ relação entre formação académica e as subescalas das necessidades, os resultados dizem que tanto as mães como os pais que não sabem ler e escrever apresentam maior preocupação relativamente as necessidades de informação, apoio, serviços da comunidade, financeiras. Porém, quanto ao funcionamento familiar, as mães diferem dos pais, sendo as mães que não sabem ler e escrever que mais sentem esta necessidade. Estes resultados corroboram o estudo de Sampaio \& Geraldes, (2006), que aponta as famílias com baixa escolarização como as que têm necessidades significativamente mais elevadas, dados os poucos recursos para responder aos enormes desafios aportados pela deficiência. Na relação entre formação acadêmica e as subescalas do IRF apurou-se que tanto as mães como os pais com o ensino superior são os que mais demostram apoiar os seus filhos nas subescalas "compreender os seus pontos fortes, as necessidades e as capacidades do seu filho", "formar sistemas de apoio" e "ter acesso à comunidade". Os resultados mostram que estas mães sentem necessidades de "conhecer os seus direitos e defenderem os interesses do seu filho", bem como "ajudar o seu filho a se desenvolver e a aprender".

Analisando a relação entre o diagnóstico funcional e as dimensões das necessidades das famílias, verifica-se que a necessidade de informação é a mais valorizada pelas famílias. No entanto, ao compararmos os diagnósticos, observa-se que as famílias de crianças com autismo apresentam o indicador mais elevado em todas as necessidades, exceto necessidades financeiras, sendo esta mais sentida pelas famílias de crianças com deficiências múltiplas. Sampaio e Geraldes, (2006) também referem que as necessidades das famílias variam de acordo com as características específicas da deficiência, e a literatura ressalta, em geral, a relevância da percepção em termos de necessidades específicas, e não globais, diante da complexidade de cada deficiência.

$\mathrm{Na}$ relação entre o grau de dependência e as necessidades que preocupam as famílias, constatou-se que as famílias de crianças com dependência motora apresentam mais necessidades de informação, apoio, explicar aos outros e financeiras do que serviços da comunidade e funcionamento familiar.

\section{Conclusões}

Os dados obtidos permitem-nos apontar para dois tipos de conclusões: um relativo à construção de serviços de IP e outro sobre as necessidades das famílias que procuram apoio para os seus filhos com transtornos do desenvolvimento. 
http://dx.doi.org/10.5902/1984686X67416

Quanto ao primeiro, a forma como serviços de IP podem ser concebidos e implementados numa perspectiva moderna, multisectorial e centrada na família, vemos que os sistemas a construir devem estar de acordo com as necessidade desenvolvimentais das famílias e as suas competências. Numa perspectiva tradicional, de estimulação, os serviços disponibilizados, de tipo terapêutico e reabilitativo, tendiam a estar pré-definidos em função da patologia/ transtorno e dos programas pré-estabelecidos, independentemente das características, cultura, funcionamento e competências da família. Até porque esta não era chamada para o centro do processo, sendo mais frequentemente vista como obstáculo. Por outro lado, uma perspectiva centrada na família exige que serviço e profissionais apostem na promoção das competências dos pais/mães, no seu desenvolvimento pessoal e emocional, e no seu emponderamento. Desta forma, o conhecimento das necessidades dos pais/mães e a forma como avaliam o resultado da sua intervenção, fornecem subsídios para a criação do serviço de IP que a instituição pretende implementar. O estudo efetuado aponta uma metodologia de trabalho com vista à criação de respostas de IP que pode ser replicada. No futuro, o IRF permitirá também avaliar o impacto do novo serviço.

O estudo apresentado permite-nos, por outro lado, compreender que as preocupações das famílias se centram especialmente no futuro, na saúde da criança e em saber como promover o seu desenvolvimento. Vemos que são as necessidades de informação e financeiras as que mais inquietam as famílias, assim como as dificuldades de acesso a serviços adequados. Todas têm necessidades de informação, sendo este um dos fatores que provoca mais estresse; a falta de informações concretas sobre o como lidar com a deficiência gera, no meio familiar, níveis elevados de ansiedade, preocupação e instabilidade e é especialmente impeditiva do seu envolvimento e empoderamento. Podemos também concluir que quanto mais as famílias se preocupam com as necessidades, menos capazes se sentem de apoiar os filhos no seu processo de desenvolvimento. É importante destacar que as necessidades são diferentes para cada família, levando em consideração as suas crenças e cultura, além das demais variáveis pessoais e sociais.

A identificação das necessidades e o estabelecimento de prioridades são elementos cruciais para a intervenção que visa fortalecer o núcleo familiar e o papel parental na promoção do desenvolvimento precoce. Para implementação de uma verdadeira prática centrada na família, torna-se imprescindível identificar as necessidades e preocupações das famílias. Após a definição dessas necessidades e prioridades, importa que os pais se 
http://dx.doi.org/10.5902/1984686X67416

autonomizem face aos serviços, mantendo estreita colaboração, mas não criando uma relação de dependência. Será fundamental para que a inclusão seja efetivada na sua plenitude, que essas crianças com deficiência se constituam cidadãos com todos os direitos e deveres.

Estes resultados apontam para a importância de conceber serviços e programas que respondam às necessidades das famílias e que possam ser efetivamente eficazes para as crianças. Acreditamos que este estudo trouxe contributos para a implementação de estratégias para melhorar o serviço prestado às famílias de crianças com deficiência, especificamente as que frequentam a AAPPE.

\section{Referências}

ALMEIDA, I. C. A intervenção centrada na família e na comunidade: $O$ hiato entre as evidências e as práticas. Análise Psicológica, 1(XXIX), 5-25, 2011.

BAILEY, D. Issues and perspetives on family assessment. Infants and Young Children, 4(1), 26-34, 1991

BAILEY, D. B.; SIMEONSSON, R. J. Assessing needs of families with handicapped infants. The Journal of Special Education, 22(1), 117-127. 1988

BAILEY, D. B.; WOLERY, M. Teaching infants and preschoolers with disabilities. New York. Macmillan. 1992

BAILEY, D. B.; BLASCO, P. M.; SIMEONSSON, R. J..Needs expressed by mothers and fathers of young children with disabilities. American Journal of Mental Retardation, 97(1), 1-10, 1992.

BAILEY, D. B., RASPA, M., OLMSTED, M. G., NOVAK, S. P., SAM, A. M., HUMPHREYS, B. P., GUILLEN, C. Development and Psychometric Validation of the Family Outcomes Survey-Revised. Journal of Early Intervention, 33(1), 6-23, 2011

BAILEY, D.; HEBBELER, K.; OLMSTED, M.; RASPA, M.; BRUDER, M.B. (2008).

Measuring Family Outcomes Considerations for Large-Scale Data Collection in Early Intervention. Infants \& Young Children, 21, 3. p 194-206, 2008. doi:

10.1097/01.IYC.0000324549.31822.c3

BJORCK-AKESSON, E.; GRANLUND, M.; SIMEONSSON, R. J. Assessment philosophies and practices in Sweden. In GURALNICK M. J. (Org), Interdisciplinary clinical assessment of young children with disabilities. Baltimore: Paul H. Brookes, Publishing Company, 2000.

CARDOSO, C. M. F.; SEIXAS, S. R.; PISCALHO, I. A importância do envolvimento da família no desenvolvimento de uma criança com hipotonia neonatal. Revista da UIIPS Unidade de Investigação do Instituto Politécnico de Santarém, 5(1), 34-45, 2017. 
CORREIA, L. M.; SERRANO, A. M. (Org.). Envolvimento Parental em Intervenção Precoce - das Práticas Centradas na Criança às Práticas Centradas na Família. Porto: Porto Editora, 2002.

COUTINHO, M. T. B. Apoio à família e formação parental. Análise Psicológica, 1(XXI), 55-64, 2004.

CRUZ, A. I.; FONTES, F.; CARVALHO, M. L. Avaliação da satisfação das famílias apoiadas pelo PIIP: Resultados da aplicação da escala ESFIP. Lisboa: Secretariado Nacional da Reabilitação, 21, 2003.

DIAS, S. C. C.; PIMENTEL, J.S. Capacitação de pais com filhos com deficiência. Diversidade e Educação: Desafios Atuais, 274-289, 2017.

DUNST, C. J. Apoiar e Capacitar as Famílias em Intervenção Precoce: o que aprendemos? In Correia, L. M.; Serrano, A. M. (Orgs.). Envolvimento Parental em Intervenção Precoce - das Práticas Centradas na Criança às Práticas Centradas na Família. Porto: Porto Editora, 1988

DUNST, C.; TRIVETTE, C.; DEAL, A. Enabling and Empowering Families: Principles and Guidelines for Practice. Cambridge, MA: Brookline, 1988.

ESPE-SHERWINDT, M. Family-centered practice: Collaboration, competency ande evidence. Support for Learning, 23(3), 136-143, 2008.

FRANCO, V,; MELO, M.; SANTOS, G.; APOLÓNIO, A.; AMARAL, L. A National Early Intervention System as a strategy to promote inclusion and academic achievement in Portugal. Frontiers in Psychology. 8, 1137. 2017. Doi: 10.3389/fpsyg.2017.01137

FRANCO, V. Perturbações do desenvolvimento e percursos inclusivos. In VICENTE CASTRO, F.; DIAZ-DIAZ, A.; FAJARDO CALDERA, I; RUIZ FERNANDEZ, I (Org),

Psicologia y Educacion: Nuevas investigaciones. Universidad de Extremadura: Psicoex, 2005. p.313-328,

FRANCO, V. A adaptação das famílias de crianças com perturbações graves do desenvolvimento- contribuição para um modelo conceptual. INFAD - International Journal of Developmental and Educational Psychology, XXI, 2. 2009.

FRANCO, V. Idealização e re-idealização no desenvolvimento dos pais e mães das crianças com deficiência. In PARLATO-OLIVEIRA, E.; COHEN, D. (Org). $\mathbf{O}$ bebê e o outro: seu entorno e suas interações, São Paulo: Instituto Langage, 2017, p.111- 127.

FRANCO, V.; APOLÓNIO, A. Desenvolvimento, Resiliência e Necessidades das Famílias com Crianças Deficientes. Revista Ciência Psicológica, 8, 40-54. 2002

FRANCO, V.; APOLÓNIO, A. Impacto da Intervenção Precoce na informação, inclusão social e funcionamento das famílias de crianças com e sem perturbações de desenvolvimento. INFAD - International Journal of Developmental and Educational Psychology, XX, 1(4), 593-604, 2008. 
GALLAGHER, J. J.; BECKMAN, P.; CROSS, A. H. Families of handicapped children: Sources of stress and its amelioration. Exceptional children, 50(1), 10-19, 1983.

GINÉ, C. Las necesidades de la familia a lo largo del ciclo vital. In. ALONSO, V; ÁNGEL M. (Org.), Familias Y Discapacidad Intelectual. Madrid: Confederación Española de Organizaciones en Favor de las Personas com Retraso Mental, 2000.

GONÇALVES, M.; SIMÕES, C. Práticas de intervenção precoce na infância - as necessidades das famílias de crianças com necessidades educativas especiais. Gestão e desenvolvimento, 17(18), 157-174, 2010.

GUALDA, D. S.; BORGES, L.; CIA, F. Famílias de crianças com necessidades educacionais especiais: recursos e necessidades de apoio. Revista Educação Especial, 26(46), 307-330, 2013.

MAHONEY, G., \& PERALES, F. (2013). The role of parents of children with Down syndrome and other disabilities in early intervention. In RONDAL, J., PERARA, J., SPIKER, D. (Org.). Neurocognitive Rehabilitation of Down Syndrome-Early Years .Cambridge University Press, 2013. p. 205-223.

MCWILLIAM, R. A.; YOUNG, H. J.; HARVILLE, K. Therapy services in early intervention: Current status, barriers, and recommendations. Topics in Early Childhood Special Education, 16(3), 348-374, 1996.

MEDINA-GOMEZ, B.; FRANCO, V. En el momento del diagnóstico son prioritarias las necesidades de los hijos con Síndrome X Frágil. Siglo Cero - Revista Española sobre Discapacidad, 49(3), no 267. pp 95-108, 2018.

MORGANHO, P. A. B. "Todos Diferentes, Todos Pais": Um Programa de Educação Parental para famílias de crianças com Autismo. Viseu: Instituto Politécnico de Viseu Escola Superior de Educação, 2015

PAVÃO, M. R.; GUALDA, D. S.; CIA, F.; SANTOS, L. S.; CHRISTOVAM, A. C. C. Rotina e necessidades de apoio: relato de familiares de crianças de zero a dois anos, público alvo da Educação Especial. Revista Educação Especial, 31(61), 447-462, 2018.

\section{PEREIRA, F. As representações dos professores de educação especial e as} necessidades das famílias. Lisboa: Secretariado Nacional de Reabilitação, 1996.

PIMENTEL, J. S. Avaliação de programas de intervenção precoce. Análise Psicológica, 1(XXII), 43-54, 2004.

PIMENTEL, J. S. Intervenção focada na família: desejo ou realidade. Perceções de pais e profissionais sobre as práticas de apoio precoce a crianças com necessidades educativas especiais e suas famílias. Lisboa: Secretariado Nacional de Reabilitação e Integração de Pessoas com deficiência. 2005

RAMíREZ, G. Á. Estudio sobre las necesidades de las familias de personas con discapacidad. Madrid: Ministerio de Sanidad, Servicios Sociales e Igualdad. 2015. 
SÁ, S. M. P.; RABINOVICH, E. P. Compreendendo a família da criança com deficiência física. Rev. Bras. Crescimento Desenvolv. Hum, 16(1), 68-84, 2006.

SAMPAIO, F. M.; GERALDES, S. Necessidades das famílias de crianças com deficiência: Um estudo com a escala Family Needs Survey. Revista da Faculdade de Ciências Humanas e Sociais, 3, 277-286, 2006.

SERRANO, A. M. Redes Sociais de Apoio e sua Relevância para a intervenção Precoce. Porto: Porto Editora, 2008.

SIEBES, R. C.; KETELAAR, M.; GORTER, J. W.; ALSEM, M.; JONGMANS, M. J. Needs of Families with Children Who Have a Physical Disability: A Literature Review. Critical Reviews in Physical and Rehabilitation Medicine, 24(1-2), 85-108, 2012.

SIMÕES, M. I. P. G.; MESQUITA, H. F. P. Necessidades das famílias de crianças com deficit cognitivo e motor. Revista de Educação Especial e Reabilitação, 17, 47-58, 2010.

SOQUEIRO, M. C.; CARVALHO, O.; MARTINS, R. M. F.; PASCOINHO, J. C. Necessidades das famílias em processos de intervenção precoce: um estudo com famílias e profissionais. Revista Diálogos e Perspetivas em Educação Especial, 4(2), 99-112, 2017.

SOUSA, H. P. M; PISCALHO, I. Contributos da intervenção precoce na rede de apoio à criança com cegueira. Interações 41, 95-120, 2016.

TURNBULL, A. P. \& TURNBULL, H. R. Families, professionals and exceptionality: A special partnership. Columbus: Charles E. Merril Publishing, 1990. 